\title{
O atendimento psicológico no Centro de Referência Especializado da Assistência Social e a visão de operadores do direito e conselheiros tutelares
}

\author{
Psychological assistance provided by a Specialized Center \\ of Reference in Social Assistance and the perspective \\ of law professionals and child protective agents
}

\author{
Suane Pastoriza FARAJ ${ }^{1}$ \\ Aline Cardoso SIQUEIRA ${ }^{1}$ \\ Dorian Mônica ARPINI ${ }^{1}$
}

\begin{abstract}
Resumo
Este estudo, de delineamento qualitativo, objetivou conhecer a percepção dos operadores do direito e conselheiros tutelares sobre o atendimento psicológico ofertado à criança e ao adolescente no Centro de Referência Especializado da Assistência Social. Através de entrevistas semiestruturadas, constatou-se que esse centro tem se constituído como um serviço de referência no encaminhamento e atendimento psicológico oferecido às vítimas de violência. $O$ atendimento do profissional da Psicologia no âmbito da Assistência Social é considerado importante para a escuta de crianças e adolescentes em situação de violência. No entanto, o atendimento oferecido pelo município nem sempre contempla a demanda existente no local e, na maioria das vezes, é prestado de forma tardia. Dessa forma, ainda se faz necessário mais investimento dos gestores municipais na implantação de serviços e contratação de profissionais especializados para atender a população infanto-juvenil em situação de violência.
\end{abstract}

Palavras-chave: Aconselhamento; Adolescente; Criança; Serviços de assistência social; Violência.

\begin{abstract}
This is a qualitative study aimed at analyzing the perception of child protective agents and law professionals about the psychological care provided to children and adolescents by a Specialized Center of Reference in Social Assistance. Semi-structured interviews were conducted, and the results indicated that this Center has been a reference in providing services such as referrals and psychological assistance to victims of violence. The assistance provided by the Psychology professionals in the context of social assistance is considered very important for the understanding and support of

$\nabla \nabla \nabla$

1 Universidade Federal de Santa Maria, Departamento de Psicologia, Programa de Pós-Graduação em Psicologia. R. Marechal Floriano Peixoto, 1750, $3^{\circ}$ andar, Sala 300, 97015-372, Santa Maria, RS, Brasil. Correspondência para/Correspondence to: S.P. FARAJ. E-mail: <suanef@yahoo.com.br>.

Artigo baseado na dissertação de S.P. FARAJ, intitulada: "A notificação da violência, o atendimento psicológico e a rede de proteção da criança e do adolescente: o olhar de profissionais do Sistema de Garantia de Direitos”. Universidade Federal de Santa Maria, 2014.
\end{abstract}


children and adolescents in a situation of violence. However, the municipal public assistance offered does not always meet local demand, and, most of the time, it starts too late. Therefore, the results show need for more local government's investments to promote the implementation of services and to hire specialized professionals to assist children and adolescents in a situation of violence.

Keywords: Counseling; Adolescent; Child; Social services; Violence.

$\mathrm{Na}$ atualidade brasileira, a violência representa um problema social que viola os direitos de muitas crianças e adolescentes. $O$ atendimento psicossocial a essa população em situação de violência é preconizado pelo Estatuto da Criança e do Adolescente (ECA) - Lei 8.069 de 13 de julho de 1990 (Brasil, 1990) - e, muitas vezes, se faz necessário devido às implicações do fenômeno no desenvolvimento social, físico e psíquico dos sujeitos, assim como na dinâmica familiar dos mesmos (Habigzang, Azevedo, Koller, \& Machado, 2006). Nesse sentido, as políticas públicas voltadas às crianças, aos adolescentes e às famílias em situação de violência constituem uma possibilidade da oferta de atendimento especializado, o qual possibilita a elaboração das vivências de situações de violência e a garantia dos direitos da população infanto-juvenil.

A atual política pública de Assistência Social tem disponibilizado serviços de proteção social especial para indivíduos e famílias que tiveram seus direitos violados, através dos Centros de Referência Especializados da Assistência Social (CREAS). Devido à complexidade do fenômeno, os CREAS são formados por equipes compostas por profissionais de diversas áreas do conhecimento, dentre elas o Serviço Social, o Direito, a Pedagogia e, sobretudo, a Psicologia (Almeida \& Goto, 2011; Macedo et al. (2011); Yamamoto \& Oliveira, 2010).

A atuação do psicólogo no âmbito da Assistência Social, em especial nos CREAS, tem sido objeto de discussões e estudos (Almeida \& Goto, 2011; Faraj \& Siqueira, 2012; Miron \& Guareschi, 2013; Yamamoto \& Oliveira, 2010). O Conselho Federal de Psicologia, através do Centro de Referência em Políticas Públicas (CREPOP), vem desenvolvendo pesquisas sobre a atuação dos psicólogos nas políticas públicas brasileiras e lançando diversos instrumentos os quais visam subsidiar as ações desenvolvidas por esse profissional na proteção social espe- cial de média complexidade (Conselho Federal de Psicologia, 2009, 2012).

A recente publicação realizada pelo Conselho Federal de Psicologia (2012) trouxe uma reflexão acerca da prática dos psicólogos nos CREAS. Destacou que a atuação da Psicologia no âmbito da Assistência Social vem sendo construída através de uma crítica reflexão acerca do seu papel e de sua potencialidade. Apontou ainda que, "a participação da Psicologia nas políticas públicas deve ser pautada na garantia dos direitos humanos, na emancipação humana, na cidadania e a serviço das lutas contra as injustiças, pobreza e violência" (p.48). Dessa forma, "não deve reforçar a tutela do Estado, a visão da caridade e a submissão dos sujeitos às políticas públicas" (p.48). Assim, o respeito aos direitos humanos, à democracia, à emancipação e à autonomia dos sujeitos são princípios os quais devem nortear a prática do psicólogo.

Os documentos que versam sobre a temática ressaltam que, nos CREAS, a prática desse profissional deve ter como base os objetivos preconizados no Guia de Orientações Técnicas do CREAS (Brasil, 2005), ou seja, deve estar voltada para o fortalecimento dos vínculos familiares e sociais, potencialização dos recursos para a superação da situação vivenciada e proteção da criança e do adolescente. Nesse sentido, o atendimento psicológico deve estar inserido na atenção psicossocial, onde é operacionalizado através de procedimentos técnicos especializados os quais visa, à estruturação das ações de atendimento e proteção à criança e ao adolescente, possibilitando a reparação e a superação da vivência de situações de violência (Conselho Federal de Psicologia, 2009, 2012).

$\mathrm{O}$ atendimento psicossocial configura-se, conforme o Conselho Federal de Psicologia (2009, p.49), como um "conjunto de atividades e ações psicossocioeducativas, de apoio especializado, desenvolvidas individualmente e em pequenos grupos 
(prioritariamente), de caráter disciplinar e interdisciplinar, de cunho terapêutico". Deve ser planejado em equipe, a partir do plano individual. O foco da Psicologia não é o atendimento psicoterápico, mas sim o psicossocial. Este pode e deve ter efeitos terapêuticos, mas não tem a mesma intervenção e objetivos da psicoterapia. Dessa forma, a atuação em equipes multidisciplinares configura uma atividade frequente do psicólogo no âmbito da Assistência Social, principalmente no CREAS (Conselho Federal de Psicologia, 2009, 2012).

Além disso, a articulação em rede também é uma prática do profissional de Psicologia no âmbito da assistencial social, sobretudo no CREAS. A atuação do psicólogo nesses centros deve estar articulada com o trabalho de profissionais inseridos em outras políticas sociais (saúde, educação, segurança, entre outras) e, principalmente, com atores os quais atuam nos órgãos de defesa de direitos (Brasil, 2005). Nessa articulação em rede, destaca-se o contato constante que o psicólogo deve ter com o Conselho Tutelar, Poder Judiciário, Ministério Público e Delegacias Especializadas. A articulação possibilita a efetivação dos objetivos estabelecidos no plano de atendimento, bem como o acesso das crianças, adolescentes e familiares em outros serviços, programas e órgãos e, principalmente, a defesa dos direitos das crianças e adolescentes (Conselho Federal de Psicologia, 2012).

Assim, este estudo teve como objetivo conhecer a percepção dos operadores do direito e conselheiros tutelares acerca do atendimento psicológico ofertado à criança, ao adolescente e seus familiares no âmbito do CREAS, a partir de suas práticas profissionais. É importante ressaltar que se entende por percepção a maneira como os profissionais analisam, avaliam e qualificam o atendimento psicológico ofertado no CREAS à população infanto-juvenil.

\section{Método}

\section{Participantes}

Trata-se de um estudo qualitativo realizado junto à Delegacia de Proteção à Criança e ao Ado- lescente, ao Ministério Público e a três Conselhos Tutelares de uma cidade do interior do Rio Grande do Sul. Esses órgãos foram incluídos no estudo por comporem o Sistema de Garantia de Direitos (SGD) da Criança e do Adolescente e atuarem no recebimento e investigação das situações de violência envolvendo essa população (Brasil, 2004). Nesse sentido, devem estar articulados com outros órgãos, instituições e serviços, especialmente com os de proteção social especial, como o CREAS.

Participaram cinco profissionais, dentre eles um delegado de polícia, um promotor de justiça e três conselheiros tutelares. O critério de inclusão adotado foi ser promotor de justiça atuante na Promotoria Especializada de Defesa da Infância e Juventude ou delegado de polícia da Delegacia de Proteção à Criança e ao Adolescente ou conselheiro tutelar no exercício da função de coordenador. No momento do estudo, dois conselheiros tutelares tinham formação acadêmica; um era formado em História e o outro em Pedagogia com especialização em Psicopedagogia. Quanto aos operadores do Direito, um tinha mestrado em Direito e o outro era especialista na área da infância e juventude pela Escola Superior do Ministério Público.

O tempo de atuação dos profissionais nos respectivos órgãos variou de dois a 17 anos. $\mathrm{Na}$ apresentação dos resultados, optou-se por não identificar o sexo e a atividade profissional do entrevistado em função dos cuidados éticos e da garantia do anonimato dos participantes, considerando as especificidades da cidade quanto à população e profissionais inseridos no SGD. Dessa forma, os entrevistados foram identificados da seguinte maneira: P1, P2, P3, P4 e P5.

\section{Instrumentos}

Foram realizadas entrevistas semiestruturadas com questões acerca do conhecimento dos profissionais sobre o atendimento psicológico oferecido pelo município à criança e/ou adolescente em situação de violência; também foi investigada a percepção e perspectivas do entrevistado acerca 
desse tipo de atendimento. As entrevistas foram realizadas individualmente no período de janeiro a abril de 2013, tendo uma duração de, aproximadamente, uma hora; cada um dos órgãos investigados ofereceu uma sala apropriada para a condução dessa etapa. No momento da entrevista, foi estabelecido o rapport e, em seguida, foram esclarecidos os objetivos da pesquisa, sendo apresentado aos profissionais o Termo de Consentimento Livre e Esclarecido, o qual foi lido e posteriormente assinado pelos mesmos.

As entrevistas realizadas foram gravadas e transcritas na íntegra. A análise foi feita utilizando a análise de conteúdo (Bardin, 1977/2011), que se refere a um conjunto de instrumentos metodológicos aplicados aos discursos, através do qual se analisam as comunicações, decompondo-as e elencando categorias.

Em um primeiro momento, realizou-se uma análise detalhada de cada entrevista individualmente e, depois, procedeu-se a análise da totalidade do material obtido, considerando a frequência, a força discursiva e a repetição. Buscou-se, dessa forma, pontos de aproximação entre as entrevistas, bem como aspectos divergentes.

A pesquisa foi respaldada pela Resolução $n^{\circ}$ 466/2012 do Conselho Nacional de Saúde (Brasil, 2012) e Resolução n 016/2000 do Conselho Federal de Psicologia (2000), as quais regulam a pesquisa com seres humanos. Inicialmente, foi realizado um contato inicial com a Delegacia de Proteção à Criança e ao Adolescente, com o Ministério Público e com os Conselhos Tutelares, a fim de apresentar os objetivos do estudo. Após uma autorização prévia de cada órgão, o projeto foi submetido, para apreciação e avaliação, ao Comitê de Ética em Pesquisa (CEP) da Instituição de Ensino Superior à qual as pesquisadoras estão vinculadas. Com a aprovação do CEP via Plataforma Brasil (protocolo n 04974412.4.0000.5346), em 10 de julho de 2012, os profissionais foram convidados a participar do estudo mediante esclarecimentos éticos.

\section{Resultados e Discussão}

\section{Centros de Referência Especializados da Assistência Social: referência no encaminhamento de crianças e adolescentes em situação de violência}

Através do estudo foi possível identificar que, entre os procedimentos realizados pelos operadores do direito e conselheiros tutelares após o conhecimento de uma situação de violência, estava o encaminhamento da criança, adolescente e família para o atendimento psicológico, como pode ser observado na fala: "... . Seja qual for a violência, a gente primeiramente ouve o que ocorreu, o fato que ocorreu com aquela criança, aquele adolescente e dá os encaminhamentos necessários... o encaminhamento para a rede de atendimento... pra receber um atendimento psicológico..." (P2). Esses dados estão de acordo com os achados de outros estudos, os quais mostraram que os órgãos de defesa dos direitos, entre eles, o Conselho Tutelar, o Juizado da Infância e Juventude e o Ministério Público, realizam o encaminhamento da criança e do adolescente em situação de violência para os serviços de referência, que abarcam o atendimento do profissional de Psicologia (Dobke, Santos, \& Dell'Aglio, 2010; Habigzang et al., 2006; Milani \& Loureiro, 2008; Santos, Costa, \& Silva, 2011).

O Centro de Referência Especializado de Assistência Social foi identificado pelos profissionais como um serviço de referência no encaminhamento e atendimento dos casos envolvendo suspeita ou situação de violência. Conforme os profissionais, "A questão da violência sempre a gente encaminha pro CREAS né, porque é um serviço de referência" (P3); "... . Violência mais especificamente, encaminha para o CREAS que é o órgão que trabalha com a questão da Psicologia, pra trabalhar a questão da demanda da violência" (P2); "... . Encaminha a vitima para o CREAS né que vai fazer todo o trabalho psicológico... " (P4). Os encaminhamentos para esse tipo de serviço, segundo os profissionais que participaram do estudo, são realizados através de um ofício ou documento, no qual consta a numeração 
do Código Internacional de Doenças (CID-10) com a seguinte legenda: 74.0 Negligência e abandono, 74.1 Sevícias físicas, 74.2 Abuso sexual, 74.3 Abuso psicológico e 74.9 Síndrome não especificada de maus tratos (Conselho Federal de Psicologia, 2012; Faraj \& Siqueira, 2012). O modelo do documento visa manter o sigilo das informações.

Destaca-se que, após o encaminhamento ao atendimento psicológico, os operadores do direito e os conselheiros tutelares mencionaram que continuavam acompanhando a criança, o adolescente e sua família, o que parece ser de extrema importância para a efetivação dos casos. A partir do estudo, pode-se identificar que os entrevistados compartilhavam da concepção expressa por Azambuja (2011), ou seja, embora nem todas as crianças que vivenciaram situação de violência apresentem problemas emocionais, em todas as situações é imprescindível avaliar e realizar o encaminhamento para o acompanhamento se este constituir uma medida necessária.

\section{Atendimento psicossocial nos Centros de Referência Especializados da Assistência Social}

Pôde-se constatar que o encaminhando da criança e/ou adolescente em situação de suspeita ou de violência, bem como das respectivas famílias ao serviço especializado (CREAS) estava relacionado à percepção dos operadores do direito e conselheiros tutelares acerca da importância desse tipo de serviço, especialmente por constituir um espaço em que é possível falar sobre a situação vivenciada e, assim, receber apoio e proteção dos órgãos competentes e superar a situação de violação de direitos. Observou-se ainda que o encaminhamento, na visão dos entrevistados, estava relacionado à possibilidade de evitar a revitimização desses indivíduos. Isso pode ser averiguado através da fala:

... . Ela é ouvida aqui, se ela quiser falar. Isso é muito importante, por que a busca pela justiça, a busca pela verdade... . Não pode revitimizar a criança e o adolescente, né. Por que a criança não se sente, o adolescente não se sente preparado pra falar sobre esse fato que violou o seu direito, ele não é ouvido aqui. Então elas saem daqui com um ofício pra ser acompanhado lá pelo psicólogo. E lá, no tempo dela, ela vai falar (P4).

O estudo realizado por Faraj e Siqueira (2012) apontou a importância do CREAS para o atendimento à criança, ao adolescente e às respectivas famílias, na medida em que busca a minimização dos danos da violência, bem como o rompimento e o enfrentamento da situação. Nessa mesma perspectiva, Arpini, Siqueira e Savegnago (2012) apontaram que os Centros de Atendimento Especializados em Vítimas de Violência podem possibilitar a elaboração das situações vivenciadas e a redução da repetição de eventos violentos. De acordo com Pacheco e Malgarim (2011), o serviço prestado pelo CREAS é imprescindível às vítimas de violência e suas famílias, pois as intervenções através dos recursos técnicos da Psicologia e da Assistência Social podem possibilitar o acesso aos conflitos gerados pelas vivências abusivas e proporcionar condições para que a família se reorganize frente às situações geradas pela violência. As autoras destacaram que a implementação desses espaços de atenção para atender a demanda dos casos de violência possibilita, a partir do acolhimento e atendimento, o fortalecimento dos vínculos, o desenvolvimento de estratégias de enfrentamento da violência e a proteção à vítima e seus familiares.

Além da atenção à criança e ao adolescente, os profissionais apontaram a importância do serviço oferecer o atendimento aos pais e/ou responsáveis e à família, para que as questões as quais envolvem a violência possam ser resolvidas e superadas. É importante ressaltar que o atendimento à família é preconizado pela política de Assistência Social (Brasil, 2004), portanto os serviços de proteção especial devem acolhê-las e atendê-las quando em situação de risco social. $\mathrm{O}$ apoio às mães, por exemplo, é importante, pois podem apresentar sentimento de impotência por não conseguirem proteger o(a) filho(a), bem como de culpa, ambivalência, entre outros. No entanto, como apontou Dell'Aglio, Moura e Santos (2011), o foco de atenção dos serviços ainda são as vítimas. As autoras salientaram ainda que o acompanhamento e a orientação às 
mães/cuidadoras influencia o tratamento dos filhos e possibilita que ofereçam apoio às crianças/adolescentes, aliviando o sofrimento. Dessa forma, faz-se necessário que os serviços proporcionem o atendimento às famílias, visto que têm um papel fundamental na superação da violência e na proteção da criança/adolescente.

Outro dado significativo apontado pelos operadores do direito e conselheiros tutelares refere-se à necessidade da oferta de um atendimento psicológico imediato à criança e ao adolescente em situação de violência no CREAS, como pode ser averiguado nas falas: "Pra nós isso é uma coisa imediata, que tem que ser feito imediato... essa é uma demanda que tem que ser atendida imediatamente..." (P3); "... . Não pode ter lista de espera. Tem que ser atendido... conforme a necessidade vai aparecendo, a demanda vai aparecendo tem que ser atendido. Então, a gente luta pra que não tenha fila de espera" (P5). Os participantes deste estudo verbalizaram que, muitas vezes, o atendimento não ocorre de maneira imediata, principalmente em função da grande demanda do município e dos poucos profissionais existentes para atendê-la e, dessa forma, muitos acabam em uma lista de espera durante semanas ou meses.

Destaca-se que tanto o atendimento quanto sua prioridade são estabelecidos pelo Estatuto da Criança e do Adolescente (Brasil, 1990) como um direito da população infanto-juvenil. Almeida e Goto (2011) apontaram a estratégia de implantar um plantão psicológico no CREAS, através da modalidade de intervenção em crise. Essa estratégia visa uma atuação breve, prioriza as condições de emergência e urgência social e, assim, acolhe e proporciona uma escuta imediata aos sujeitos os quais tiveram seus direitos violados. Essa modalidade de intervenção poderia ser incluída em outros CREAS a fim de possibilitar a garantia do atendimento imediato à população em outros municípios.

Os profissionais entrevistados também apontaram que estão fiscalizando e cobrando dos gestores municipais a oferta desse atendimento especializado e imediato. Um profissional destacou que a criação de uma segunda unidade do CREAS no de órgãos da defesa dos direitos. Nesse sentido, ficou evidente que o CREAS representa para os operadores do direito e conselheiros tutelares um serviço de referência no atendimento às crianças e aos adolescentes em situação de violência.

\section{Interação entre os atores da rede de proteção}

Os instrumentos os quais orientam a prática do psicólogo no âmbito do CREAS apontam que as intervenções no âmbito da Psicologia devem estar articuladas e integradas a outras políticas públicas e aos órgãos que compõem o Sistema de Garantia de Direitos, dentre eles, os Conselhos Tutelares, o Ministério Público e as Delegacias Especializadas (Conselho Federal de Psicologia, 2009, 2012). A partir dos relatos de operadores do direito e conselheiros tutelares, pôde-se identificar que os órgãos de defesa dos direitos interagem com o psicólogo do CREAS:

... . A gente tem contato diariamente com o CREAS... (P2);

... . Os técnicos [psicólogos] entram em contato com a gente ou por telefone ou a gente marca reunião, vai até o serviço... (P3);

... . Vou ter acesso ao parecer psicológico que as psicólogas vão enviar. E esse parecer, que além de assim, ter toda a evolução dela como paciente, tem a conclusão do psicó$\log 0$ (P4)

As falas acima mostram dois tipos de atuação envolvendo os psicólogos do CREAS e os atores dos órgãos de defesa dos direitos: uma voltada para um trabalho articulado, a partir de contatos telefônicos e reuniões de discussão de caso, envolvendo, assim, trocas de conhecimentos, informações e intervenções conjuntas; a outra, realizada através de envio e leitura de relatórios, parece envolver uma menor articulação. É importante considerar que ações articuladas e integradas compreendem o recebimento e o encaminhamento dos casos, acesso aos prontuários e processos judiciais. Abarcam, ainda, a discussão dos casos por todos os profissionais envolvidos no atendimento, bem 
como a troca de saberes e experiências (Lorencini, Ferrari, \& Garcia, 2002). Dessa forma, as atuações devem ser realizadas através de reuniões, nas quais possam se estabelecer trocas de informações, discussão de casos e intervenções, não se restringindo ao envio e leitura de relatórios acerca do atendimento psicológico.

Sabe-se que o trabalho em rede não é fácil nem simples, pois implica superar uma prática realizada durante muitos anos de maneira fragmentada e desarticulada. Assim, estudos vêm apontando a necessidade do fortalecimento da rede de atendimento e proteção à criança e ao adolescente em situação de violência (Faraj \& Siqueira, 2012, Habigzang et al., 2006). Ações em rede possibilitam o bem-estar desses indivíduos na medida em que promove a saúde, a segurança, a proteção e também a busca dos direitos (Pelisoli, Pires, Almeida, \& Dell'Aglio, 2010).

Dessa forma, parece importante que os conselheiros tutelares, delegados e promotores tenham clareza do seu papel, limites e possibilidades de intervenção nos casos de violência, assim como conhecimento do funcionamento da rede de atendimento, pois a integração de órgãos e serviços é fundamental na proteção à criança, ao adolescente e às famílias em suspeita ou situação de violência. Do mesmo modo, é fundamental o psicólogo compreender o seu papel nos casos de violação de direitos, bem como realizar a articulação em rede com profissionais de outros serviços de atendimento e, principalmente, órgãos de defesa dos direitos, a fim de garantir os direitos da criança e do adolescente preconizados no ECA (Conselho Federal de Psicologia, 2009; Pinheiro \& Fornari, 2011). O Conselho Federal de Psicologia (2012) destaca que é um compromisso ético do psicólogo no CREAS colaborar para a articulação das instituições que compõem o SGD.

\section{0 atendimento psicológico: percepções e perspectivas}

A maioria dos profissionais entrevistados considerou o trabalho do profissional da Psicologia satisfatório. Segundo uma participante, "Essa parte da rede, ela tá funcionado muito bem... . Eu considero o trabalho a contento" (P4). No entanto, a carência de serviços especializados e profissionais da Psicologia que atendam a criança e o adolescente em situação de violência foi apontada pelos profissionais: "... . Ainda faltam serviços e faltam profissionais. Isso o município ainda tá pecando" (P3). Dessa forma, os entrevistados assinalaram a preocupação com relação à gestão do serviço especializado, relatando a grande demanda dos casos e os poucos profissionais existentes para atendê-la. A instabilidade dos profissionais do CREAS, ocasionada por contratos temporários do órgão municipal, também foi identificada como uma preocupação dos conselheiros tutelares e operadores do direito, pois esta é sentida como prejudicial no atendimento das crianças e adolescentes. A grande demanda de casos de violência, a carência de recursos humanos e materiais, a troca constante de profissionais e o pouco investimento dos gestores municipais também são apontados em outros estudos sobre o assunto (Conselho Federal de Psicologia, 2012; Faraj \& Siqueira, 2012; Macedo et al., 2011; Pedersen, 2010).

Quanto às perspectivas em relação ao atendimento psicológico, os profissionais entrevistados disseram acreditar que o psicólogo deve oferecer apoio psicológico às crianças, adolescentes e às suas respectivas famílias, possibilitando o rompimento da violência, a minimização dos sintomas decorrentes dessa situação e a superação do trauma. Isso pode ser observado nos relatos:

A gente espera pelo menos... que amenize um pouco aquela situação de violência ou que cesse também aquela situação de violência (P2);

... . A minha expectativa é que amenize ou até mesmo sane a questão do trauma... (P3);

... . A gente espera que aquele atendimento possa trazer algum benefício pessoal pra ela (criança), que ela consiga melhorar o comportamento, o relacionamento que ela tem com a família, com a escola, com a comunidade onde ela vive né (P5).

A atuação do psicológico no âmbito da Assistência Social visa o desenvolvimento de atividades 
especializadas, de caráter psicossocioeducativo, apoio e orientação realizadas de maneira individual ou em grupos (Conselho Federal de Psicologia, 2009). Dentre as atividades que podem ser desenvolvidas pelo psicólogo no CREAS, destacam-se: acolhida, acompanhamento psicossocial, entrevista, visita domiciliar, intervenções grupais e articulação em rede (Conselho Federal de Psicologia, 2012). A atuação deve estar voltada para uma atenção psicossocial que acontece na troca cotidiana dos profissionais os quais integram a equipe. Estes devem ter o compromisso de compartilhar e estabelecer responsabilidades viabilizando a soma de conhecimento e o estabelecimento de estratégias para a efetividade das intervenções. Assim, o profissional da Psicologia e a equipe do CREAS devem buscar, através das ações desenvolvidas, amenizar os danos causados pela violência, bem como a interrupção do seu ciclo e a possibilidade de reconstrução dos vínculos afetivos (Conselho Federal de Psicologia, 2009, 2012).

Nesse sentido, parece que há uma expectativa, por parte de alguns profissionais entrevistados, além das possibilidades de trabalho do Psicólogo no CREAS. A superação do trauma requer um trabalho aprofundado, não contemplado pela atuação esperada do psicólogo no CREAS. Cabe, portanto, aos próprios profissionais os quais atuam no atendimento das vítimas de violência estarem atentos às demandas desses pacientes e encaminhá-los para serviços especializados quando necessário; isso porque o atendimento no CREAS não deve se constituir como um processo de psicoterapia (Brasil, 2005; Conselho Federal de Psicologia, 2009). Dessa forma, deve existir no município serviços específicos que ofereçam o atendimento psicoterápico para atender essa demanda.

A identificação da situação de violência e a elaboração de relatório também foram apontadas como tarefas esperadas pelos operadores do direito e conselheiros tutelares no âmbito do atendimento psicossocial. A perspectiva de um dos profissionais é de "Que o serviço identifique né, que o serviço encaminhe pra nós, pra gente tomar as medidas ou encaminhe pra delegacia pra ser afastado o agressor ou pra ser até punido o agressor..." (P3).
Outro entrevistado mencionou, "... . Que esse atendimento chegue no resultado, essa criança foi ou essa criança não foi abusada. É isso que eu espero..." (P5). A produção de relatórios, assim como a averiguação de denúncias no âmbito da prática do profissional de Psicologia no CREAS, ainda carece de discussões (Conselho Federal de Psicologia, 2012). No entanto, considera-se que o "resultado final" não cabe ao psicólogo, mas sim aos órgãos competentes - Ministério Público, Varas Criminais, Juizados da Infância e Juventude, Delegacias -, os quais devem avaliar se ocorreu ou não um crime, se a criança teve ou não os seus direitos violados. Em relação aos relatórios, quando necessários, devem ser elaborados de acordo com o preconizado pela Resolução $n^{\circ}$ 007/2003 do Conselho Federal de Psicologia, que instituiu o Manual de Elaboração de Documentos Escritos produzidos pelo psicólogo (Conselho Federal de Psicologia, 2003). Deve conter, ainda, somente as informações extremamente necessárias para a tomada de decisões e adoção de medidas de proteção, priorizando o bem-estar e a segurança da criança/adolescente, previstos no Código de Ética do psicólogo (Conselho Federal de Psicologia, 2005).

A atuação desse profissional no CREAS é uma prática que vem sendo construída e ainda requer debates a respeito dos limites e das possibilidades no âmbito da Assistência Social (Conselho Federal de Psicologia, 2012). Mas, sendo o foco da Psicologia nesses centros o atendimento psicossocial, este deve ser realizado de maneira interdisciplinar, articulado com outras políticas e, principalmente, com os órgãos de defesa dos direitos. Nesse sentido, de acordo com Pinheiro e Fornari (2011), o psicólogo inserido na rede de proteção deve exercer o papel de "intermediário", compreendendo as vivências dos pacientes e desencadeando os mecanismos de proteção que competem a outras instâncias através da notificação do caso e/ou pareceres psicológicos. Não compete ao psicólogo medidas legais, assim como não compete aos profissionais de outras áreas do conhecimento a avaliação psicológica, a qual permite a identificação dos danos emocionais e sua gravidade diante de uma situação de violência, bem como o tratamento psicológico em si. Dessa forma, "será no cruzamento de saberes que se produzirá a proteção" (Pinheiro \& Fornari, 2011, p.308). 


\section{Considerações Finais}

O Centro de Referência Especializado da Assistência Social se destacou como um serviço de referência no encaminhamento e atendimento às vítimas de violência, pois, segundo os participantes, este oferecia ações no âmbito da Psicologia. $O$ atendimento do psicólogo foi percebido como satisfatório pela maioria dos entrevistados, no entanto, aspectos importantes quanto ao tempo para conseguir o atendimento foram apontados. Dessa forma, parecia haver uma demanda, no momento da realização do estudo, que excedia a capacidade de atendimento disponível nos serviços do município. Assim, verificou-se a necessidade de mais investimentos dos gestores municipais na contratação continuada de profissionais capacitados para que o serviço possa ofertar um atendimento especializado e imediato às crianças, adolescentes e familiares.

A partir deste estudo, foi possível identificar que alguns atores do SGD tinham uma expectativa que extrapolava os limites e as possibilidades de atendimento no âmbito do CREAS. Além disso, percebeu-se que ainda se faz necessário o fortalecimento do trabalho em conjunto entre psicólogos, conselheiros tutelares e operadores do direito. Assim, deve-se ampliar as articulações dos segmentos da rede através de reuniões, discussão de casos e ações, a fim de possibilitar a resolução e a efetivação das ações nos casos de violação de direitos envolvendo as crianças e os adolescente do município. Nessa área de atuação, é fundamental a presença de psicólogos capacitados para o atendimento psicossocial e para a articulação da rede.

Destaca-se que ainda existem muitos desafios no atendimento à criança e ao adolescente tanto no âmbito da atuação da Psicologia quanto na prática dos órgãos de defesa dos direitos. Apesar da legislação ter preconizado o atendimento psicossocial à criança e ao adolescente em situação de violência no ano de 1990, este ainda está sendo construído na prática dos profissionais envolvidos com a temática, bem como na atuação em rede.

Dessa maneira, a atuação dos psicólogos nos CREAS e nos outros serviços de atendimento que compõem o sistema público de saúde são desafios os quais ainda demandam muitos esforços e investimentos. Este estudo traz uma importante contribuição ao tema, embora algumas limitações possam ser apontadas, como, por exemplo, a escolha dos participantes, os quais representam um pequeno número de profissionais do SGD. Também pode ser apontado o fato da pesquisa ter contemplado apenas um município do estado do Rio Grande do Sul; considerando a pluralidade e as profundas diferenças socioculturais e econômicas presentes na realidade brasileira, estudos em outros contextos podem apresentar resultados diferentes. Nesse sentido, sugere-se o olhar dos próprios psicólogos, bem como da população possam ser contemplados em pesquisas futuras, ampliando e qualificando o fazer psicológico e a rede de atendimento.

\section{Colaboradores}

Todos os autores contribuíram na concepção e desenho do estudo, análise de dados e redação final.

\section{Referências}

Almeida, B. P., \& Goto, T. A. (2011). Intervenção e cuidado com crianças e adolescentes vitimizadas: atuação do psicólogo no Programa Sentinela (CREAS) em Poços de Caldas (MG). Mudanças: Psicologia da Saúde, 19(1-2), 89-98.

Arpini, D. M., Siqueira, A. C., \& Savegnago, S. D. O. (2012). Trauma psíquico e abuso sexual: o olhar de meninas em situação de vulnerabilidade. Psicologia: Teoria e Prática, 14(2), 88-101.

Azambuja, M. R. (2011). Inquirição da criança vítima de violência sexual: proteção ou violação de direitos? Porto Alegre: Livraria do Advogado.

Bardin, L. (2011). Análise de conteúdo. Lisboa: Edições 70. (Originalmente publicado em 1977).

Brasil. Ministério do Desenvolvimento Social e Combate a Fome. (2005). Guia de Orientação Técnica - SUAS $n^{\circ}$ 1: proteção social básica de assistência social. Brasília: Autor. Recuperado em outubro 16, 2012, de http://www.recria.org.br/quemcompoe/governa mentais/fas/guia

Brasil. Ministério da Saúde. (2012). Resolução n 466, de 12 de dezembro de 2012. Diário Oficial da União, n 12, jun. 2013, Seção 1, p.59.

Brasil. Presidência da República. (1990). Lei n 8.069, de 13 de julho de 1990. Dispõe sobre o Estatuto da 
Criança e do Adolescente e dá outras providências. Brasília: Autor.

Brasil. Presidência da República. (2004). Guia escolar: método para identificação de sinais de abuso e exploração sexual de crianças e adolescentes. Brasília: Autor.

Conselho Federal de Psicologia. (2000). Resolução CFP $\mathrm{n}^{\circ}$ 016/2000, de 20 de dezembro de 2000. Ementa: dispõe sobre a realização de pesquisa em Psicologia com seres humanos. Brasília: Autor.

Conselho Federal de Psicologia. (2003). Resolução n $007 /$ 2003. Institui o Manual de Elaboração de Documentos Escritos produzidos pelo psicólogo, decorrentes de avaliação psicológica e revoga a Resolução CFP $n^{\circ}$ 17/2002. Brasília: Autor.

Conselho Federal de Psicologia. (2005). Resolução CFP $n^{\circ}$ 010/05. Aprova o código de ética profissional do psicólogo. Recuperado em julho 8, 2013, de http:// www.pol.org.br

Conselho Federal de Psicologia. (2009). Serviço de proteção social a crianças e adolescentes vítimas de violência, abuso e exploração sexual e suas famílias: referências para a atuação do psicólogo. Brasília: Autor. Recuperado em junho 10, 2013, de http://crepop. pol.org.br

Conselho Federal de Psicologia. (2012). Referências técnicas para prática de psicólogas(os) no Centro de Referência Especializado da Assistência Social - CREAS. Brasília: Autor.

Dell'Aglio, D. D., Moura, A., \& Santos, S. S. (2011). Atendimento a mães de vítimas de abuso sexual e abusadores: considerações teóricas e práticas. Psicologia Clínica, 23(2), 53-73.

Dobke, V. M., Santos, S. S., \& Dell'Aglio, D. D. (2010). Abuso sexual intrafamiliar: da notificação ao depoimento no contexto processual-penal. Temas em Psicologia, 18(1), 167-176.

Faraj, S. P., \& Siqueira, A. C. (2012). O atendimento e a rede de proteção da criança e do adolescente vítima de violência sexual na perspectiva dos profissionais do CREAS. Barbarói, 37, 67-87.

Habigzang, L. F., Azevedo, G. A., Koller, S. H., \& Machado, P. X. (2006). Fatores de risco e de proteção na rede de atendimento a crianças e adolescentes vítimas de violência sexual. Psicologia: Reflexão e Crítica, 19(3), 379-386.

Lorencini, B. D. B., Ferrari, D. C. A., \& Garcia, M. R. C. (2002). Conceito de redes. In D. C. A. Ferrari \& T. C.
C. Vecina (Eds.), O fim do silêncio na violência familiar (pp.298-310). São Paulo. Ágora.

Macedo, J. P., Souza, A. P., Carvalho, D. M., Magalhães, M. A., Souza, F. M. S., \& Dimenstein, M. (2011). O psicólogo brasileiro no SUAS: quantos somos e onde estamos? Psicologia em Estudo, 16(3), 479-489.

Milani, R. G., \& Loureiro, S. R. (2008). Famílias e violência doméstica: condições psicossociais pós ações do conselho tutelar. Psicologia: Ciência e Profissão, 28(1), 50-67.

Miron, A. X., \& Guareschi, N. M. F. (2013). A Psicologia na política de Assistência Social: movimentos de uma experiência de gestão no CREAS. In L. R. Cruz, L. Rodrigues, \& N. M. F. Guareschi (Eds.), Interlocuções entre a Psicologia e a Política Nacional de Assistência Social (pp.118-30). Santa Cruz do Sul: EDUNISC.

Pacheco, M. L., \& Malgarim, B. G. (2011). Centro de Referência Especializado de Assistência Social: apanhados teóricos sobre uma rede especial de apoio e proteção em casos de abuso sexual infantil. Revista de Psicologia da IMED, 3(2), 545-553.

Pedersen, J. R. (2010). Abuso sexual intrafamiliar: do silêncio ao seu enfrentamento (Dissertação de mestrado não-publicada). Pontifícia Universidade Católica do Rio Grande do Sul, Porto Alegre.

Pelisoli, C., Pires, J. P. M., Almeida, M. E., \& Dell'Aglio, D. D. (2010). Violência sexual contra crianças e adolescentes: dados de um serviço de referência. Temas em Psicologia, 18(1), 85-97.

Pinheiro, L., \& Fornari, V. L. (2011). O papel do psicólogo nos casos de violência contra a criança e o adolescente. In M. R. F. Azambuja \& M. H. M. Ferreira (Eds.), Violência sexual contra crianças e adolescentes (pp.298-317). Porto Alegre: Artmed.

Santos, V. A., Costa, L. F., \& Silva, A. X. (2011). As medidas protetivas na perspectiva de famílias em situação de violência sexual. Psico, 42(1), 77-86.

Yamamoto, O. H., \& Oliveira, I. F. (2010). Política social e Psicologia: uma trajetória de 25 anos. Psicologia: Teoria e Pesquisa, 26(N. Esp.), 9-24.

Recebido: abril 16, 2014

Versão final: abril 7, 2015

Aprovado: abril 16, 2015 